\title{
A Belief-based Decision-making Framework for Spectrum Selection in Cognitive Radio Networks
}

\author{
J. Pérez-Romero, Member, IEEE, A. Raschellà, O. Sallent, and A. Umbert, Member, IEEE
}

\begin{abstract}
This paper presents a comprehensive cognitive management framework for spectrum selection in cognitive radio networks. The framework uses a belief vector concept as a means to predict the interference affecting the different spectrum blocks and relies on a smart analysis of the scenario dynamicity to properly determine an adequate observation strategy to balance the trade-off between achievable performance and measurement requirements. In this respect, the paper shows that the interference dynamics in a given spectrum block can be properly characterized through the second highest eigenvalue of the interference state transition matrix. Therefore, this indicator is retained in the proposed framework as a relevant parameter to drive the selection of both the observation strategy and spectrum selection decision-making criterion. The paper evaluates the proposed framework to illustrate the capability to properly choose among a set of possible observation strategies under different scenario conditions. Furthermore, a comparison against other state-of-the-art solutions is presented.
\end{abstract}

Index Terms - Belief Vector, Cognitive Radio, Spectrum Selection, Testbed.

\section{INTRODUCTION}

$\mathrm{T}_{\mathrm{s}}^{\mathrm{H} \cos }$ HE Cognitive radio (CR) has emerged in recent years as a paradigm that envisages an intelligent radio able to utomatically adjust its behavior based on the active monitoring of its environment [1]. From a general perspective, $\mathrm{CR}$ employs a cognitive cycle that involves observations of the environment, analysis of these observations, decision making to intelligently configure certain radio parameters and finally execution of the decisions via actions. Analysis and decision can be supported by learning mechanisms that exploit the knowledge obtained from the execution of prior decisions. This general approach can be applied to efficiently and adaptively modify different radio operational parameters, such as the frequency, transmit power, and modulation scheme. Thus, the CR concept as a means to achieve a smart and efficient operation of wireless networks has applicability in a variety of different scenarios. For example, it has been

Manuscript submitted on August $27^{\text {th }} 2014$ and accepted on November $29^{\text {th }}$ 2015.

Copyright (c) 2015 IEEE. Personal use of this material is permitted. However, permission to use this material for any other purposes must be obtained from the IEEE by sending a request to pubs-permissions@iee.org.

J. Pérez-Romero, O. Sallent and A. Umbert are with the Department of Signal Theory and Communications, Universitat Politècnica de Catalunya (UPC), Barcelona, Spain. (e-mail: \{jorperez, sallent, annau\}@tsc.upc.edu).

A. Raschellà is with the School of Computing \& Mathematical Sciences, Liverpool John Moores University (LJMU), Liverpool, UK (e-mail: a.raschella@ljmu.ac.uk). identified as a promising solution to solve the trade-off between spectrum demand growth and spectrum underutilization by enabling the development of dynamic spectrum access (DSA) mechanisms [2], [3]. Similarly, the use of the cognitive cycle is also applicable in the area of selforganizing networks (SONs), where it is envisaged as a means of performing an automated optimization of the network parameters [4]. Moreover, with the increased complexity associated with future networks, the introduction of intelligence into the network will be an important requirement for the smart management of $5^{\text {th }}$-generation (5G) networks [5], and $\mathrm{CR}$ concepts are expected to play a relevant role.

One of the key elements in enabling the efficient operation of the cognitive cycle in CR networks is the acquisition of the relevant knowledge about the radio environment that drives the decision-making processes. For that purpose, the observation stage typically involves making measurements at several nodes of a CR network. These measurements must be reported to the entity in charge of analyzing them to extract the relevant information about the radio environment and to execute the decision making. This task is typically accomplished through signaling procedures supported by cognitive control channels [6], [7]. Given that the observation stage can be costly in terms of practical requirements, such as signaling overhead and battery consumption, decision-making strategies able to efficiently operate with a minimum number of measurements are of high interest for enhancing $C R$ operation.

In this context, different decision-making tools for spectrum selection have been proposed in the literature with the aim of predicting the future behavior of the interference and to operate with reduced measurement needs. A multichannel spectrum selection algorithm that predicts the duration of the spectrum holes based on the prior statistics of the channels to limit the interference generated to a primary network was recently proposed in [8]. In [9], an opportunistic spectrum access scheme is presented based on estimating the probability of a channel appearing idle. A survey of tools that have been used in the literature for spectrum prediction can be found in [10]. In this respect, approaches based on hidden Markov models (HMMs) and multilayer perceptron are used in [11], whereas partial periodic pattern mining (PPPM) is used in [12]. Furthermore, game theory is used in [13] for spectrum selection in the context of a carrier aggregation framework. The applicability of game theory to opportunistic spectrum access is discussed in [14] and the references therein, where the need for acquiring knowledge regarding the statistical 
behavior of the radio environment is identified as a key requirement. Regarding the acquisition of information about the radio environment, several research works rely on partially observable Markov decision processes (POMDPs) [15] that combine partial observations of the radio environment at specific periods of time with a statistical characterization of the system dynamics. In this direction, [16] and [17] propose opportunistic spectrum access approaches for channels that can be either busy or idle, assuming a single unlicensed user. In [18], the problem is studied for a multi-user scenario through a collaborative approach. The use of POMDPs for spectrum selection in CR networks is proposed in [19] and [20]. In [21], the restless multi-armed bandit problem is used to find the optimal policy of sensing channels to maximize the expected throughput. In [22], a classification of decisionmaking techniques is given depending on their a priori knowledge and their sensitivity to sensing errors. The effects of imperfect sensing on spectrum access are also investigated in [23]. In [24], the authors introduce the belief-based decision-making concept for spectrum selection and analyze the trade-off between performance and observation requirements, whereas the concept is assessed experimentally in [25].

Building on the above foundations, this paper presents a comprehensive cognitive management framework for spectrum selection in CR networks based on the belief vector concept as a means to characterize and predict the environmental dynamics. The belief vector assesses the probability that the radio environment is under specific conditions (e.g., interference levels) at a certain time based on past measurements. As long as the belief vector predicts the existing conditions at the time the decision is made with sufficient accuracy, proper decisions can be made with minimum requirements in terms of observations.

This paper substantially expands the previous works of the authors [24], [25] in several facets. The main contributions of this paper are as follows: (1) In terms of cognitive network architecture, general belief-based decision making is integrated into a complete functional model that jointly considers the spectrum selection and determination of the most suitable observation strategy. (2) In terms of cognitive cycle stages, a smart characterization of the scenario dynamicity is proposed through the use of the second-highest eigenvalue of the state transition matrix as a key representative metric of the interference dynamics in a spectrum block. (3) In terms of cognitive algorithmic solutions and based on the newly proposed eigenvalue-based metric, a new scheme for selecting the observation strategy and associated spectrum selection decision-making criterion to balance the trade-off between performance and measurement requirements is developed. (4) The proposed framework is first evaluated through simulations to illustrate its behavior under different scenarios and compare it against other references from the literature. Second, an experimental evaluation via a real-time testbed is also carried out to assess some practical aspects related to the dynamic variations of the interference sources.

The remainder of the paper is organized as follows. Section
II presents the system model and general spectrum selection decision-making criterion based on the belief vector concept. Section III presents the proposed functional architecture to implement the spectrum selection framework and provides the details of each component, including the characterization of the scenario dynamism based on the eigenvalue analysis of the state transition matrices. Section IV presents the performance evaluation via simulations and a testbed. Concluding remarks are provided in Section V.

\section{System Model and SPectrum Selection Decision- MAKING CRITERION}

The system model considered in this paper assumes a set of $j=1, \ldots, L$ radio links, each intended to support data transmission between either a pair of terminals or between a terminal and infrastructure node. The $j$-th radio link will support a certain data service characterized by a required bit rate $R_{\text {req, } j}$ and will generate data transmission sessions of a certain duration $D_{j}$.

The focus of this work will be on the spectrum selection functionality that determines the spectrum to be assigned to each radio link. For this purpose, it is assumed that the $L$ radio links are controlled by a cognitive management entity in charge of spectrum selection decisions. The spectrum is organized in a set of $i=1, \ldots, M$ spectrum blocks (SBs), each characterized by a central frequency and bandwidth. The SBs can span different spectrum bands subject to different interference conditions and different regulatory regimes. The spectrum selection process will ensure that there is no internal interference among the $L$ radio links, so the interference sources in each SB are assumed to be other external transmitters out of the control of the cognitive management entity. It is assumed that these external transmitters have the same rights of spectrum use as the considered radio links. This is the typical case for SBs in unlicensed bands. Similarly, for SBs in bands where the shared use of the spectrum is allowed under the primary/secondary sharing model, such as television white spaces (TVWS), it is assumed that there are mechanisms in place (e.g., through contacting a geolocation database) to identify the SBs that are available for secondary use. In this case, the radio links managed by the cognitive management entity correspond to secondary users. In turn, the external transmitters that may cause interference are other secondary devices that do not form part of the system under the control of the cognitive management entity.

Possible examples of practical applications of the proposed framework include: (i) a home networking scenario in which the radio links correspond to communicating devices (e.g., TVs, consoles, and laptops) coordinated by a Digital Home Manager, as considered in [26]; (ii) a small cell network in which some small cells opportunistically extend their capacity with additional channels coming from unlicensed bands, e.g., in Long Term Evolution - Unlicensed (LTE-U), as considered in [27]; (iii) a wireless network exploiting device-to-device (D2D) communications between specific terminals making use of additional frequencies coming from, e.g., unlicensed 
bands, as in [28].

The available bit rate $R_{j, i}$ for the $j$-th link in the $i$-th SB will depend on both the propagation conditions between the $j$-th link transmitter and receiver and the interference in the $i$-th SB experienced at the receiver. Then, the problem considered here is to perform an efficient allocation of the SBs to the radio links by properly matching the bit rate requirements with the achievable bit rate in each SB. This task will be performed by the spectrum selection decision making, which will take a socalled action, corresponding to the allocation of a SB to a radio link, anytime that a data transmission session is initiated on this radio link. The action made for the $j$-th link at time $t$ is denoted as $a_{j}(t) \in\{1, \ldots, M\}$ and corresponds to the selected SB among those currently available.

It is worth mentioning that this paper makes a distinction between the spectrum selection and the channel access functionalities. While the former decides the assignment of SBs to radio links, channel access decides when the actual transmissions are carried out on the assigned SB, so it could be used to enable that multiple radio links share the same SB in the time domain (e.g. by means of a Listen-Before-Talk mechanism as considered in [29], or by means of scheduling strategies). Since the focus of this paper is on the spectrum selection, it is assumed that a SB can only be assigned to one radio link, so no channel access functionalities are considered. Therefore, in the case that all the SBs are occupied, a new data transmission session request is blocked.

The considered interference model denotes as $I_{j, i}(t)=I_{\text {max }, j, i} \sigma_{i}(t)$ the interference spectral density measured by the receiver of the $j$-th link in the $i$-th SB. To capture the fact that interfering sources may exhibit time-varying characteristics, $\sigma_{i}(t)$ is a SB-specific term between 0 and 1 (i.e., $\sigma_{i}(t)=0$ when no interference exists, and $\sigma_{i}(t)=1$ when the interference reaches its maximum value $I_{\text {max }, j, i}$.

For modeling purposes, it is considered that the set of possible values of $\sigma_{i}(t)$ is translated into a discrete set of interference states $S^{(i)}(t) \in\{0,1, \ldots, K\}$, where state $S^{(i)}(t)=k$ corresponds to $\sigma_{k-1}<\sigma_{\mathrm{i}}(t) \leq \sigma_{k}$ for $k>0$ and to $\sigma_{\mathrm{i}}(t)=\sigma_{0}=0$ for $k=0$. Furthermore, $\sigma_{K}=1$. The interference evolution for the $i$ th block is modeled as an ergodic discrete-time Markov process with the state transition probability from being in state $k$ at time $t$ and moving to state $k^{\prime}$ in the next time step $t+1$ given by

$$
p_{k, k^{\prime}}^{(i)}=\operatorname{Pr}\left[S^{(i)}(t+1)=k^{\prime} \mid S^{(i)}(t)=k\right]
$$

Then, the state transition probability matrix for the $i$-th SB is defined as

$$
\mathbf{P}^{(\mathbf{i})}=\left[\begin{array}{cccc}
p_{0,0}^{(i)} & p_{0,1}^{(i)} & \cdots & p_{0, K}^{(i)} \\
p_{1,0}^{(i)} & p_{1,1}^{(i)} & & p_{1, K}^{(i)} \\
\vdots & & \ddots & \vdots \\
p_{K, 0}^{(i)} & p_{K, 1}^{(i)} & \cdots & p_{K, K}^{(i)}
\end{array}\right]
$$

Without loss of generality, the time axis is assumed to be discretized in time steps. It is assumed that the state of the $i$-th
SB, $S^{(i)}(t)$, evolves independently from the other SBs. In addition, because the interference comes from external sources, it is assumed that the cognitive management entity does not have any control on the state evolution of this external interference. Besides, it is also assumed that the external interference is non-responsive, i.e. that it does not change as a reaction to the actions taken by the cognitive management entity.

Moreover, let us define $\boldsymbol{\pi}^{(\mathrm{i})}=\left[\begin{array}{llll}\pi_{0}^{(i)} & \pi_{1}^{(i)} & \cdots & \pi_{K}^{(i)}\end{array}\right]^{\mathrm{T}}$, where superscript $\mathrm{T}$ denotes the transpose operation, as the steadystate probability vector. $\pi_{k}^{(i)}$ is the probability that the $i$-th SB is in the $k$-th interference state.

Each radio link with a data session in course (referred to as an active link) will obtain a reward that measures the obtained performance depending on the interference state of the allocated SB at each time. The reward is a metric between 0 and 1 that captures the suitability of the allocated SB for the $j$ th radio link, depending on the bit rate that can be achieved with respect to the required bit rate $R_{\text {req,j. }} . r_{j, k}^{(i)}$ denotes the reward that the $j$-th link obtains upon using its allocated SB $i$ when the interference state is $S^{(i)}(t)=k$. The reward vector of the $j$-th link in the different interference states of the $i$-th SB is $\mathbf{r}_{\mathbf{j}}^{(\mathbf{i})}=\left[\begin{array}{llll}r_{j, 0}^{(i)} & r_{j, 1}^{(i)} & \cdots & r_{j, K}^{(i)}\end{array}\right]^{\mathrm{T}}$. Many possible definitions of the reward metric as a function of the bit rate may exist (e.g., sigmoid functions, linear functions, and the fittingness factor concept of [30]).

The average reward experienced by the $j$-th link in the $i$-th SB along a session starting to transmit data at time $t+1$ and ending after a certain duration of $D_{j}$ time steps is given by

$r_{\text {SESSION, }, j}^{(i)}=\frac{1}{D_{j}} \sum_{n=1}^{D_{j}} r_{j, S^{(i)}(t+n)}^{(i)}$

With all of these foundations, the spectrum selection policy executed at time $t$ for the $j$-th radio link will target the maximization of the expected reward that the session will experience along its duration:

$a_{j}(t)=\arg \max _{\substack{i \in\{1, \ldots, M\} \\ i \text { available }}} E\left[\frac{1}{D_{j}} \sum_{n=1}^{D_{j}} r_{j, S^{(i)}(t+n)}^{(i)}\right]$

where the selection is made among the subset of available SBs, i.e., those that are not allocated to any other radio link at the decision-making time $t$.

The estimation of the future evolution of the reward in each SB until the session end will exploit measurements (observations) of the interference state of the SBs carried out at specific time instants in the past, together with the statistical characterization of the interference dynamics. In particular, $o^{(i)}\left(t-m^{(i)}\right)$ denotes the observation (measurement) of the $i$-th SB that provides the value of the interference state at time step $t^{-} m^{(i)}$, i.e., $o^{(i)}\left(t-m^{(i)}\right)=S^{(i)}\left(t^{-} m^{(i)}\right)$. Then, the criterion of (4) can be reformulated as 


$$
a_{j}(t)=\arg \max _{\substack{i \in\{1, \ldots, M\} \\ i \text { available }}} \Phi_{j}^{(i)}(t)
$$

where $\Phi_{j}^{(i)}(t)$ is the SB-dependent decision function to be maximized, given by

$$
\Phi_{j}^{(i)}(t)=\frac{1}{\overline{D_{j}}} \sum_{n=1}^{\overline{D_{j}}} E\left[r_{j, S^{(i)}(t+n)}^{(i)} \mid o^{(i)}\left(t-m^{(i)}\right)=S^{(i)}\left(t-m^{(i)}\right)\right]
$$

Given that the session duration $D_{j}$ will typically be random and unknown at the decision-making time $t$, it has been characterized statistically in (6) in terms of its average value $\overline{D_{j}}$. In (6), the estimation of the expected reward achieved in the $i$-th $\mathrm{SB}$ at future time instants $t+n$ based on the past observation at $t^{-} m^{(i)}$ will rely on the statistical characterization of the interference dynamics given by the socalled belief vector. It is defined as $\mathbf{b}^{(i)}(t)=\left[\begin{array}{llll}b_{0}^{(i)}(t) & b_{1}^{(i)}(t) & \cdots & b_{K}^{(i)}(t)\end{array}\right]^{\mathrm{T}}$, where component $b_{k}^{(i)}(t)$ is the conditional probability that the $i$-th $\mathrm{SB}$ will be in state $S^{(i)}(t)=k$ at time $t$ given the last observation of the actual interference state that was taken at time step $t^{-} \mathrm{m}^{(i)}$, that is

$$
b_{k}^{(i)}(t)=\operatorname{Pr}\left[S^{(i)}(t)=k \mid o^{(i)}\left(t-m^{(i)}\right)=S^{(i)}\left(t-m^{(i)}\right)\right]
$$

Then, the expected reward obtained in the $i$-th SB at time $t+n$ can be expressed in terms of the belief vector as

$$
E\left[r_{j, S^{(i)}(t+n)}^{(i)} \mid o^{(i)}\left(t-m^{(i)}\right)=S^{(i)}\left(t-m^{(i)}\right)\right]=\mathbf{b}^{(\mathbf{i}) \mathbf{T}}(t+n) \mathbf{r}_{\mathbf{j}}^{(\mathbf{i})}
$$

By making use of (8), the decision function (6) can be expressed in terms of the belief vector as

$$
\Phi_{j}^{(i)}(t)=\frac{1}{\overline{D_{j}}} \sum_{n=1}^{\overline{D_{j}}} \mathbf{b}^{(\mathbf{i}) \mathbf{T}}(t+n) \mathbf{r}_{\mathbf{j}}^{(\mathbf{i})}
$$

\section{FUNCTIONAL ARCHITECTURE TO IMPLEMENT THE PROPOSED SOLUTION}

To implement the spectrum selection decision-making criterion given by (5) and (9), a belief-based spectrum selection (BBSS) framework is proposed. The BBSS framework consists of a centralized cognitive management entity, whose functional architecture is shown in Figure 1.

The proposed architecture is composed of two main entities, namely, the decision-making entity and knowledge management entity. The decision-making entity involves two decision processes: the spectrum selection decision making (SSDM), which is responsible for determining the spectrum to be assigned to each link and implements the decision-making criterion given by (5) and (9), and the observation strategy decision making (OSDM), which selects the observation strategy to specify the time instants when measurements have to be carried out in each SB. The decision-making entity is supported by the knowledge management entity, which collects, processes and stores the necessary information from the environment to drive the decision-making process. For that purpose, it includes three main components: the knowledge database (KD) stores different statistics and measurements characterizing the interference state of the available SBs; the knowledge manager (KM) processes the statistics and measurements contained in the $\mathrm{KD}$ to extract the relevant information that will support the decision-making process; and the $\mathrm{KD}$ acquisition is in charge of triggering the collection of measurements to fill the KD and to verify the correctness of the stored information to address changes in the stationary conditions of the environment.

The decision-making and knowledge management entities rely on the control and context awareness (CA) blocks to support their operation. Specifically, the control block provides the signaling mechanisms to communicate with the different nodes of the network, and the CA block supports the knowledge management by performing measurements (observations) of the interference state of the different SBs following the observation strategy selected by the OSDM. In the following, a detailed description of the processes carried out at the different entities is provided.

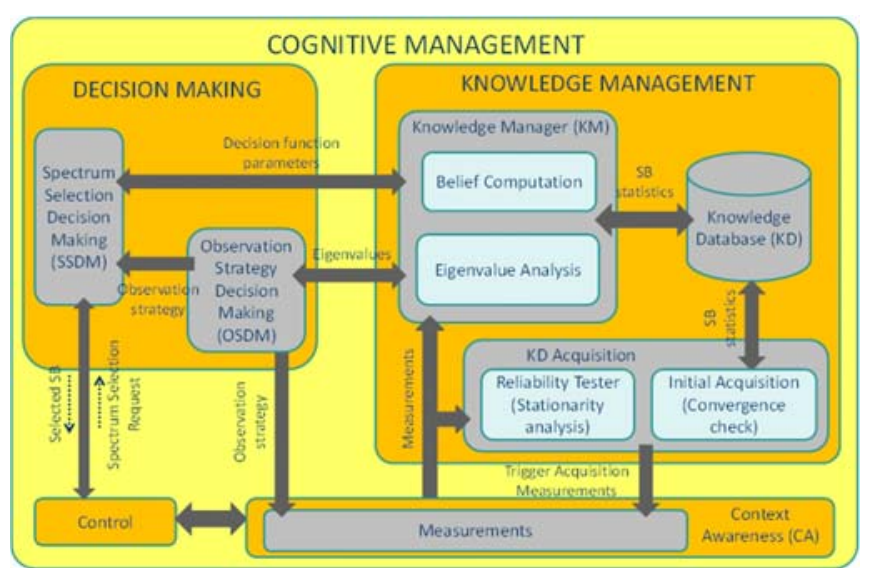

Fig.1 Proposed functional architecture of the BBSS framework.

\section{A. Knowledge management entity}

The knowledge management entity collects and processes the relevant information of the environment to drive the decision-making process. As a result, it is based on the characterization of the SBs in terms of their interference states and associated rewards. It consists of the following components:

\section{1) Knowledge Database}

The KD stores the following information about the operational environment: (1) State transition probability matrix $\mathbf{P}^{(i)}$ for the different SBs; (2) Steady-state probabilities $\boldsymbol{\pi}^{(\mathbf{i})}$ for the different SBs; (3) Reward vectors $\mathbf{r}_{\mathbf{j}}^{(\mathbf{i})}$ with the values of the reward that the different radio links can obtain in each SB for each interference state; (4) Most recent observation $o^{(i)}\left(t-m^{(i)}\right)=S^{(i)}\left(t^{-} m^{(i)}\right)$ of the actual interference state of the $i$-th SB that was measured at time step $t^{-} m^{(i)}$.

\section{2) Knowledge Manager}

The knowledge manager is in charge of the belief computation and eigenvalue analysis functionalities.

\subsection{Belief Computation}

This module analyzes and processes the information 
retained in the $\mathrm{KD}$ to extract the relevant knowledge that will be used by the spectrum selection decision-making process. In particular, it will be responsible for keeping the belief vector values for each SB updated and providing the result to the decision-making entity each time that a new spectrum selection has to be made. The computation of the belief vector of the $i$-th SB at a certain time instant $t$ is performed recursively, starting from the last observation of the actual interference state that was taken at time step $t^{-} m^{(i)}$. Specifically, considering that $o^{(i)}\left(t^{-} m^{(i)}\right)=S^{(i)}\left(t^{-} m^{(i)}\right)$, the components of the belief vector at time $t^{-} \mathrm{m}^{(i)}$ are given by

$b_{k}^{(i)}\left(t-m^{(i)}\right)=\left\{\begin{array}{crr}1 & \text { if } & k=S^{(i)}\left(t-m^{(i)}\right) \\ 0 & \text { otherwise }\end{array}\right.$

This is expressed in vector notation as

$\mathbf{b}^{(\mathbf{i})}\left(t-m^{(i)}\right)=\mathbf{x}\left(S^{(i)}\left(t-m^{(i)}\right)\right)$

where $\mathbf{x}(k)$ is defined as a column vector of $K+1$ components numbered from 0 to $K$, which are all equal to 0 , except for the $k$-th component, which is equal to 1 . The belief vector at a time instant $t>t^{-} m^{(i)}$ can be obtained from the belief vector at the previous time step $t-1$ using the state transition probability matrix as follows:

$\mathbf{b}^{(\mathbf{i}) \mathbf{T}}(t)=\mathbf{b}^{(\mathbf{i}) \mathbf{T}}(t-1) \cdot \mathbf{P}^{(\mathbf{i})}$

By recursively applying (12) for the last $m^{(i)}$ time steps and by using (11), the belief vector at time $t$ as a function of the last observation is given by

$$
\mathbf{b}^{(\mathbf{i}) \mathbf{T}}(t)=\mathbf{b}^{(\mathbf{i}) \mathbf{T}}\left(t-m^{(i)}\right)\left[\mathbf{P}^{(\mathbf{i})}\right]^{m^{(i)}}=\mathbf{x}^{\mathbf{T}}\left(S^{(i)}\left(t-m^{(i)}\right)\right)\left[\mathbf{P}^{(\mathbf{i})}\right]^{m^{(i)}}
$$

To compute the decision function (9) at time $t$, the belief vector must be extrapolated to future time instants $t+n$. This can be easily achieved from (12), leading to

$$
\mathbf{b}^{(\mathbf{i}) \mathbf{T}}(t+n)=\mathbf{b}^{(\mathbf{i}) \mathbf{T}}(t)\left[\mathbf{P}^{(\mathbf{i})}\right]^{n}
$$

\subsection{Eigenvalue Analysis}

The accuracy of the prediction of the future interference state in the $i$-th SB at time $t+n$, given by the belief $\mathbf{b}^{(\mathbf{i}) \mathbf{T}}(t+n)$, will depend on the total time elapsed since the last measurement $o^{(i)}\left(t-m^{(i)}\right)$ on this SB, i.e., $n+m^{(i)}$ time steps, as well as on the dynamism and randomness exhibited by the interference conditions affecting the $i$-th SB. In highly dynamic environments, where the interference level changes frequently in a random manner, if $n+m^{(i)}$ is large, one can expect that the accuracy of $\mathbf{b}^{(\mathbf{i}) \mathbf{T}}(t+n)$ will be low. Thus, as the belief is driving the decision-making process in (9), the spectrum selection may make incorrect decisions (e.g., assigning the $i$-th $\mathrm{SB}$ to a radio link expecting that the interference observed in the future will be low even though a high interference level will be observed). In contrast, for rather static environments, where the interference level remains stable for long periods of time, the accuracy of the belief will be high even when $n+m^{(i)}$ is large.

Therefore, the eigenvalue analysis module is responsible for characterizing through a proper metric the degree of dynamism of the radio environment. This characterization is used by the observation strategy to ensure that the elapsed time $n+m^{(i)}$ is adequate to make accurate decisions based on (9). To characterize the metric that defines the degree of dynamism of the interference and its impact on the belief vector computation, let us start by analyzing the belief vector evolution depending on the time $m^{(i)}$ elapsed since the last observation of a certain SB. It can be easily proven that if $m^{(i)}$ tends to infinity, i.e., there are no observations in this SB, the belief vector tends to the steady-state probability vector, that is:

$\lim _{m^{(i)} \rightarrow \infty} \mathbf{b}^{(\mathbf{i}) \mathrm{T}}(t)=\lim _{m^{(i)} \rightarrow \infty}\left[\mathbf{b}^{(\mathbf{i}) \mathbf{T}}\left(t-m^{(i)}\right)\left[\mathbf{P}^{(\mathbf{i})}\right]^{m^{(i)}}\right]=\boldsymbol{\pi}^{(\mathbf{i}) \mathbf{T}}$

The proof of (15) is a straightforward application of a wellknown property of ergodic discrete time Markov processes [31]. It states that for a Markov process with state transition probability matrix $\mathbf{P}^{(\mathbf{i})}$, steady-state probability vector $\boldsymbol{\pi}^{(\mathbf{i})}$ and any vector $\mathbf{y}$ representing an initial state probability distribution, the following relationship holds:

$\lim _{n \rightarrow \infty} \mathbf{y}^{\mathbf{T}}\left[\mathbf{P}^{(\mathbf{i})}\right]^{n}=\boldsymbol{\pi}^{(\mathbf{i}) \mathbf{T}}$

Then, (15) is obtained simply by considering $\mathbf{y}=\mathbf{b}^{(\mathbf{i})}\left(t^{-}\right.$ $\left.m^{(i)}\right)$. From (16), condition (15) can be expressed in terms of the future evolution of the belief vector as

$\lim _{n \rightarrow \infty} \mathbf{b}^{(\mathbf{i}) \mathrm{T}}(t+n)=\lim _{n \rightarrow \infty}\left[\mathbf{b}^{(\mathbf{i}) \mathbf{T}}(t)\left[\mathbf{P}^{(\mathbf{i})}\right]^{n}\right]=\boldsymbol{\pi}^{(\mathbf{i}) \mathbf{T}}$

The convergence speed of $\mathbf{b}^{(\mathbf{i})}(\mathrm{t})$ toward the steady state reflected in (15) and (17) will be associated with the interference dynamics in the $i$-th SB captured in the state transition matrix $\mathbf{P}^{(\mathbf{i})}$. If the interference varies slowly, it is more likely to have similar conditions at time $t+n$ to those existing at time $t$ (i.e., $\mathbf{b}^{(\mathbf{i})}(\mathrm{t})$ will be more similar to $\mathbf{b}^{(\mathbf{i})}(t+n)$ ) than if interference varies frequently and in a random manner. To capture this effect based on the properties of $\mathbf{P}^{(\mathbf{i})}$, let us consider the following theorem:

Theorem 1: The absolute value of the second-highest eigenvalue of matrix $\mathbf{P}^{(\mathbf{i})}$, denoted as $\left|\lambda_{1}^{(i)}\right|$, drives the convergence of the belief vector $\mathbf{b}^{(\mathbf{i})}(t)$ toward the steady state. A lower value of $\left|\lambda_{1}^{(i)}\right|$ will lead to a more rapid convergence.

Proof: See Appendix 1.

To illustrate Theorem 1, Figure 2 plots the evolution of the belief vector components for three different examples of state transition matrices of 2 states. The corresponding values of the second highest eigenvalue for each of the three matrices are $\lambda_{1}^{(i)}=0.9998,0.98$ and 0.8 . In all cases, the steady state probabilities are $\pi_{k}^{(i)}=0.5$ for all states. The initial belief vector at the observation time is $\mathbf{b}^{(\mathbf{i})}\left(t^{-} n\right)=\mathbf{b}^{(\mathbf{i})}(0)=[1,0]$ in all cases. Whereas the case $\lambda_{1}^{(i)}=0.9998$ exhibits a slow convergence, such that the initial value is still valid for a long time, the convergence speed increases when the value of $\lambda_{1}^{(i)}$ 
decreases. For example, when $\lambda_{1}^{(i)}=0.8$, the belief vector has converged toward the steady state probability after only a few time steps.

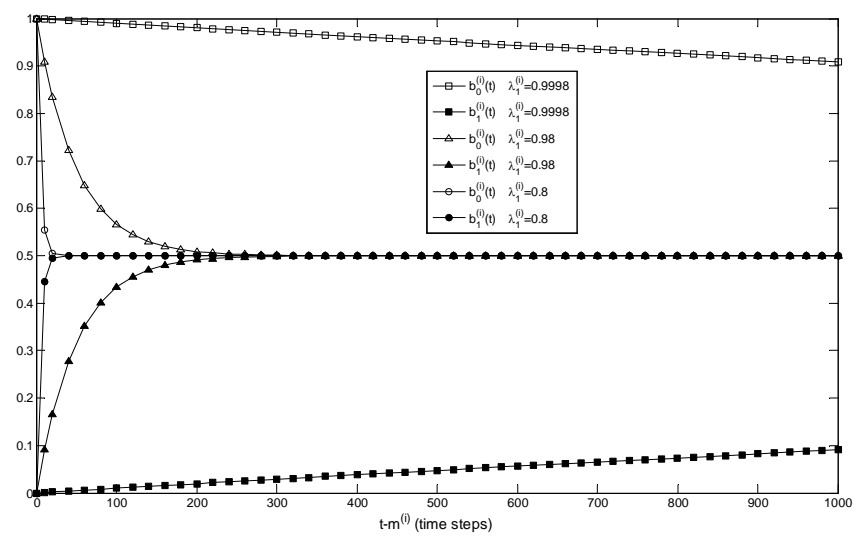

Fig.2 Examples of belief vector evolution for different values of $\lambda_{1}^{(i)}$.

Moreover, to estimate the time needed to reach convergence, the following corollary is defined:

Corollary 1: The convergence time of the belief vector $\mathbf{b}^{(\mathbf{i})}(t)$ toward the steady state can be estimated as $-1 / \ln \left|\lambda_{1}^{(i)}\right|$.

Proof: See Appendix 2.

Based on Theorem 1 and Corollary 1 , the eigenvalue analysis entity will evaluate the values of $\left|\lambda_{1}^{(i)}\right|$ for the different SBs, making use of the state transition matrices stored in the $\mathrm{KD}$, and will take them as representative values of the dynamism of the radio environment. These values will be used by the OSDM entity to select the observation strategy to be used.

\section{3) KD Acquisition}

The KD acquisition entity is responsible for filling the KD contents with the abovementioned statistics described in Section III.A.1. This involves the following two main functionalities:

\subsection{Initial Acquisition}

This functionality corresponds to the measurement acquisition process to fill the KD with the values of the state transition probability matrices $\mathbf{P}^{(\mathbf{i})}$ for the different $\mathrm{SBs}$, the steady-state probabilities $\boldsymbol{\pi}^{(\mathbf{i})}$ and the reward vectors $\mathbf{r}_{\mathbf{j}}^{(\mathbf{i})}$ that will be used during the system operation. These values will be computed by measuring the durations of the interference states in the different SBs and the associated rewards in the different radio links to obtain multiple time samples of each parameter to be acquired (e.g., the values of the state transition probabilities and steady-state probabilities). Each parameter will be estimated as the average of a sufficient number of samples that ensures convergence under certain reasonable limits. In particular, the considered convergence condition in this work is that the size of the $95 \%$ confidence interval of every measured parameter is below a fraction $\delta=0.2 \%$ of the measured average value.

\subsection{Reliability Tester (Stationarity Analysis)}

Once the acquisition phase has been completed, the resulting values of the above parameters will be kept in the
$\mathrm{KD}$, and they will be assumed to be valid as long as the stationary conditions of the environment do not change significantly with respect to the conditions existing at the time when the statistics were taken. In the case of a non-stationary environment, in which the statistical behavior of the interference may change after some time (e.g., because the interference sources have modified their positions or novel interference sources have arisen in the proximity), the system must implement mechanisms to detect these relevant changes and trigger the $\mathrm{KD}$ acquisition process again so that the statistics are properly updated. These mechanisms form part of the so-called Reliability Tester (RT) module.

Thanks to the RT, the BBSS framework is able to react in front of changes in the behavior of the interference. Specifically, when variations in the statistical behavior of the external interference occur, these are detected by the RT, which in turn triggers the regeneration of the KD.

The details of the operation of the RT module are outside the scope of this paper. As a reference, the hypothesis testing mechanism proposed in [32] to detect the changes in the interference behavior could be easily adapted to work in the framework of this paper. The capability of the BBSS framework to operate in non-stationary scenarios depends on the rate of variation of the interference, the time needed to acquire the statistical parameters of the new interference conditions and the time that the acquired interference conditions can be exploited by the cognitive management entity.

\section{B. Decision-making entity}

This entity involves the OSDM and SSDM functionalities, as detailed in the following.

\section{1) Observation Strategy Decision Making (OSDM)}

The observation strategy specifies the time instants when the actual interference state in each SB is measured. Then, the OSDM functionality is responsible for selecting the observation strategy to be applied in each SB. The observation strategy should ensure that the time $m^{(i)}$ elapsed between the last observation and SSDM execution time $t$ is adequate to compute the belief vector $\mathbf{b}^{(\mathbf{i}) \mathbf{T}}(t+n)$ and make accurate decisions based on (9). As explained in the previous section, this will be related to the interference dynamics in each SB. Therefore, the OSDM functionality will employ the eigenvalues $\left|\lambda_{1}^{(i)}\right|$ associated with each SB provided by the eigenvalue analysis function. In addition, the estimation of the belief vector $\mathbf{b}^{(\mathbf{i}) \mathbf{T}}(t+n)$ at the future instants $t+n$ also depends on the application duration $D_{j}$, which establishes the maximum time horizon $n$ over which the belief vector must be estimated. To account for this factor, the OSDM will make use of the traffic characterization in terms of the average session duration for all links $\bar{D}$ and the total average session generation rate $\rho$ (sessions/time step).

Figure 3 presents the proposed OSDM criterion to choose among the following three observation strategies:

a) Instantaneous measurements (IM) strategy: This strategy consists of performing instantaneous measurements of the interference states in all SBs at the time $t$ when a new session 
must be established, i.e., at the time when the SSDM is executed. In this specific case, the belief vector in (13) will always be computed with $m^{(i)}=0$; thus, it will capture the exact interference state at the decision-making time $t$. Then, the belief vector at $t$ will be given by

$\mathbf{b}^{(\mathbf{i}) \mathbf{T}}(t)=\mathbf{x}^{\mathbf{T}}\left(S^{(i)}(t)\right)$

b) Periodic measurements (PM) strategy: This strategy consists in performing periodic measurements of the $i$-th SB with an observation period $T_{o b s}^{(i)}$. In this manner, the elapsed time $m^{(i)}$ between the last observation of the $i$-th SB and the decision-making time $t$ will always be upper bounded by $m^{(i)} \leq T_{\text {obs }}^{(i)}$. To ensure that the measurement obtained $m^{(i)}$ time steps ago is sufficiently representative of the interference state at time $t$, it can be sufficient to ensure that the belief vector has not reached convergence during the time between two consecutive observations. According to Corollary 1, this can be achieved if the observation period fulfills the following condition:

$T_{\text {obs }}^{(i)}<\frac{1}{-\ln \left|\lambda_{1}^{(i)}\right|}$

As a further refinement of this periodic approach, it will be assumed that only the SBs that are not allocated to any link will be measured, as they are the only SBs that can be considered in the decision-making process. In turn, when a SB is released, it will also be measured in the case that the time since the last observation exceeds $T_{o b s}^{(i)}$.

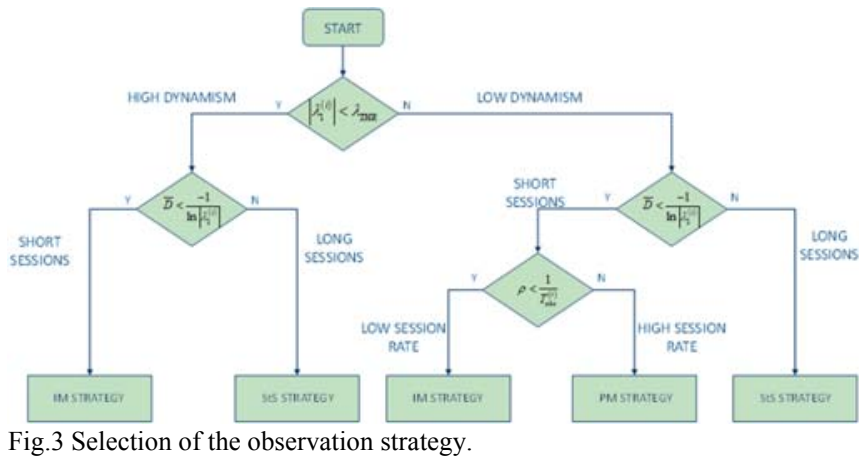

c) Steady-state (StS) strategy: This is the simple case in which no actual observations are performed. In this case, $m^{(i)} \rightarrow \infty$; thus, from (15), the values of the belief vector will be equal to the steady-state probabilities $\boldsymbol{\pi}^{(\mathbf{i}) \mathbf{T}}$.

The rationality of the observation strategy selection of Figure 3 is described in the following:

- In highly dynamic environments, i.e., $\left|\lambda_{1}^{(i)}\right|<\lambda_{\text {THR }}$, where $\lambda_{\text {IHR }}$ is a threshold to be set, the convergence of the belief vector toward the steady-state probabilities will be extremely fast, so measurements made at a given time will no longer be a valid indication of the actual interference state after a short time. In this case, two extreme approaches are considered for the observation strategy, depending on the actual needs of the decision making. If the decision making must accurately track the actual variability of the environment (e.g., this would be the case when decisions need to look only for a short-term period after the decision is made, such as when applications generate sessions with a short duration $\bar{D}$ compared to the duration $-1 / \ln \left|\lambda_{1}^{(i)}\right|$ to reach the steady state), the observation strategy would need to ensure that the time $m^{(i)}$ between the last measurement and the decision-making time is as close to 0 as possible; thus, an IM strategy that executes measurements just at the time when the decision is made (i.e., each time a session is generated) is a suitable option. In contrast, if the decision making needs to have a longer term perspective (i.e., when the session duration $\bar{D}$ is larger than the time $-1 / \ln \left|\lambda_{1}^{(i)}\right|$ needed to reach the steady state), the steadystate probabilities provide a good indication of the dynamic behavior of the interference, so measurements would not be needed. Then, the selected option is the StS strategy.

- In environments with low dynamism, i.e., $\left|\lambda_{1}^{(i)}\right| \geq \lambda_{\text {THR }}$, the convergence of the belief vector toward the steady state is slower. This means that the observations made at a certain time can be representative of the actual behavior over a longer time horizon (i.e., over a longer time $m^{(i)}$ ). Then, for session durations $\bar{D}$ shorter than the time $-1 / \ln \left|\lambda_{1}^{(i)}\right|$ needed to reach the steady state, it will be worth using previous measurements (either through IM or PM strategies) because they can be representative of the SB state along the session duration. In this case, the choice between IM and PM is related to the session arrival rate $\rho$ that reflects the rate at which the spectrum selection decision making is triggered. In particular, if the session arrival rate is high compared to the observation period $T_{o b s}^{(i)}$ (i.e., $\rho>1 / T_{o b s}^{(i)}$ ), the PM strategy becomes a suitable option because each observation made will be applicable for multiple spectrum selection decision-making processes associated with the new sessions generated between two consecutive observations. In this manner, the signaling and battery consumption associated with the measurement procedures can be substantially reduced compared to the IM that makes measurements for every new generated session. In contrast, if the session arrival rate is low (i.e., $\rho<1 / T_{o b s}^{(i)}$ ), meaning that the spectrum selection decision making will be mainly inactive between two consecutive observation periods, IM becomes a more suitable option because it only makes the measurements at the time when they are needed. Finally, in the case of long session durations (i.e., larger than the time needed to achieve the steady state $\bar{D}>-1 / \ln \left|\lambda_{1}^{(i)}\right|$ ), the average reward achieved along the session duration will be mainly driven by the steady-state behavior of the SB instead of the actual measurements at session 
initiation; thus, in this case, the simpler StS strategy becomes a suitable choice.

\section{2) Spectrum Selection Decision Making (SSDM)}

The SSDM functionality will be responsible for deciding the $\mathrm{SB}$ to be assigned each time that a new session is established in a certain radio link. For that purpose, it will implement the decision-making criterion given by (5) based on the general decision function (9). This general decision function can be particularized for each of the observation strategies selected by the OSDM as follows:

a) IM strategy: In this case, the belief vector at the decisionmaking time $t$ is given by (18). Then, by considering (18) and the estimation of the future belief vector given by (14) in the general decision-making function (9), it yields

$\Phi_{j}^{(i)}(t)=\frac{1}{\overline{D_{j}}} \mathbf{x}^{\mathbf{T}}\left(S^{(i)}(t)\right)\left(\sum_{n=1}^{\overline{D_{j}}}\left[\mathbf{P}^{(\mathbf{i})}\right]^{n}\right) \mathbf{r}_{\mathbf{j}}^{(\mathbf{i})}$

b) PM strategy: In this case, the general decision-making function (9) can be computed from the belief vector $\mathbf{b}^{(\mathbf{i}) \mathbf{T}}(t)$ at the decision-making time $t$ given by (13) and its future evolution given by (14), yielding

$$
\Phi_{j}^{(i)}(t)=\frac{1}{\overline{D_{j}}} \mathbf{b}^{(\mathbf{i}) \mathbf{T}}(t)\left(\sum_{n=1}^{\overline{D_{j}}}\left[\mathbf{P}^{(\mathbf{i})}\right]^{n}\right) \mathbf{r}_{\mathbf{j}}^{(\mathbf{i})}
$$

c) StS strategy: In this case, $m^{(i)} \rightarrow \infty$; thus, from (15), the belief vector yields $\mathbf{b}^{(\mathbf{i}) \mathbf{T}}(t) \rightarrow \boldsymbol{\pi}^{(\mathbf{i}) \mathbf{T}}$. Then, by using (14) and (15), the general decision-making function (9) becomes

$$
\begin{aligned}
& \Phi_{j}^{(i)}(t)=\lim _{m^{(i)} \rightarrow \infty} \frac{1}{\overline{D_{j}}} \mathbf{b}^{(\mathbf{i}) \mathbf{T}}(t)\left(\sum_{n=1}^{\overline{D_{j}}}\left[\mathbf{P}^{(\mathbf{i})}\right]^{n}\right) \mathbf{r}_{\mathbf{j}}^{(\mathbf{i})}=\frac{1}{\overline{D_{j}}} \boldsymbol{\pi}^{(\mathbf{i}) \mathbf{T}}\left(\sum_{n=1}^{\overline{D_{j}}}\left[\mathbf{P}^{(\mathbf{i})}\right]^{n}\right) \mathbf{r}_{\mathbf{j}}^{(\mathbf{i})}= \\
& =\frac{1}{\overline{D_{j}}}\left(\sum_{n=1}^{\overline{D_{j}}} \boldsymbol{\pi}^{(\mathbf{i}) \mathbf{T}}\left[\mathbf{P}^{(\mathbf{i})}\right]^{n}\right) \mathbf{r}_{\mathbf{j}}^{(\mathbf{i})}=\frac{1}{\overline{D_{j}}} \sum_{n=1}^{\overline{D_{j}}} \boldsymbol{\pi}^{(\mathbf{i}) \mathrm{T}} \mathbf{r}_{\mathbf{j}}^{(\mathbf{i})}=\boldsymbol{\pi}^{(\mathbf{i}) \mathbf{T}} \mathbf{r}_{\mathbf{j}}^{(\mathbf{i})}
\end{aligned}
$$

The belief computation entity in the $\mathrm{KM}$ will provide the decision making with the values of the belief vector $\mathbf{b}^{(\mathbf{i}) \mathbf{T}}(t)$, state transition probability matrices $\mathbf{P}^{(\mathbf{i})}$ and reward vector $\mathbf{r}_{\mathbf{j}}^{(\mathbf{i})}$, such that the abovementioned decision-making functions (20) to (22) can be computed in accordance with the observation strategy.

\section{Context awareness entity}

This entity is responsible for acquiring the required measurements to support the operation of the Knowledge Management entity. Specifically, it will provide the different observations $o^{(i)}(t)=S^{(i)}(t)$ that will be used by the $\mathrm{KM}$ and stored in the KD. Measurements will be triggered by the OSDM functionality in accordance with the observation strategy or by the KD acquisition functionality (e.g., at the initial acquisition or after detecting a change in the stationary conditions to fill the contents of the KD). Measurements will be delivered to the $\mathrm{KM}$ and/or the $\mathrm{KD}$ acquisition that will process them to obtain the KD statistics.

Measurements performed by the CA can be carried out at either the centralized entity or at other nodes of the different links. In this case, the CA will rely on the control entity to communicate with these nodes and retrieve the measurements.

\section{Control entity}

The control entity will provide the signaling means to support the communication between the cognitive management entity and the different nodes of the network. In the context of the spectrum selection process considered in this paper, two main functions are envisaged for this control entity:

- Whenever a new session has to be established in a given radio link, the control entity will trigger the decision making requesting the allocation of a SB and will inform the involved nodes about the result of this allocation.

- Whenever the CA needs to collect a measurement at a certain node of the network, the control entity will exchange the necessary signaling messages with this node to request and retrieve the measurement.

The signaling exchange relies on a cognitive control channel that allows for the transmission of different information elements and the realization of diverse operations within a cognitive radio system. Details on the specific implementation and signaling exchange are outside the scope of this paper. In [6] and [7], different possibilities are identified for the implementation of such a cognitive control channel, classified into radio access-independent implementations (based on, e.g., IEEE 1900.4, IEEE 802.21, or IETF PAWS) and radio access-dependent implementations (based on, e.g., 3GPP L1 and L2, IEEE 802.11, or Bluetooth).

\section{Performance Evaluation}

This section presents the performance assessment of the proposed BBSS framework. First, the evaluation is performed via system-level simulations, which allow the concept to be validated in a variety of scenarios depending on the characteristics of the interference in the different SBs and the number of radio links and associated traffic requirements. The simulation environment is also used to benchmark the proposed framework against other state-of-the-art solutions. Finally, additional results are obtained using a real-time testbed platform to better assess the practicality of the BBSS cognitive management logic when some theoretical assumptions (e.g., the Markovian behavior of the interference dynamics) do not hold.

\section{A. General scenario parameters}

This section describes the assumptions and scenario parameters that have been considered to evaluate the performance achieved by the proposed cognitive management framework via simulations. Up to $M=10$ SBs in the ISM 2.4 $\mathrm{GHz}$ and the TVWS bands are considered in the different simulations. Three interference states are considered for each SB. The detailed characteristics of each SB in terms of central frequencies, bandwidth, average durations of the interference states and corresponding values of $\lambda_{1}^{(i)}$ are presented in Table I. Two different sets of parameters are considered reflecting two degrees of dynamism in the interference variation, denoted as LD (low dynamism) and HD (high dynamism). 
TABLE I

CHARACTERISTICS OF THE SBS CONSIDERED IN THE SIMULATIONS

\begin{tabular}{|c|c|c|c|c|c|c|c|}
\hline & & $\begin{array}{l}\text { Freq. } \\
\text { (MHz) }\end{array}$ & $\begin{array}{c}\text { BW } \\
\text { (MHz) }\end{array}$ & $\begin{array}{c}S(\mathbf{i})=0 \\
\text { (time } \\
\text { steps) }\end{array}$ & $\begin{array}{c}\mathrm{S}(\mathbf{i})=1 \\
\text { (time } \\
\text { steps) }\end{array}$ & $\begin{array}{c}\mathbf{S}(\mathbf{i})=\mathbf{2} \\
\text { (time } \\
\text { steps) }\end{array}$ & $\lambda_{1}^{(i)}$ \\
\hline \multirow{5}{*}{$\begin{array}{l}\text { H } \\
\text { D }\end{array}$} & SB1,6 & $\begin{array}{l}2412 \\
2432\end{array}$ & 20 & 24 & 12 & 3 & 0.91 \\
\hline & SB2,7 & $\begin{array}{c}482 \\
498\end{array}$ & 16 & 3 & 24 & 24 & 0.94 \\
\hline & SB3,8 & $\begin{array}{l}762 \\
778\end{array}$ & 16 & 24 & 18 & 8 & 0.93 \\
\hline & SB4,9 & $\begin{array}{l}610 \\
626\end{array}$ & 16 & 12 & 12 & 8 & 0.88 \\
\hline & SB5,10 & $\begin{array}{l}2452 \\
2472\end{array}$ & 20 & 18 & 18 & 6 & 0.92 \\
\hline \multirow{5}{*}{$\begin{array}{l}\text { L } \\
\text { D }\end{array}$} & SB1,6 & $\begin{array}{l}2412 \\
2432\end{array}$ & 20 & 480 & 360 & 120 & 1 \\
\hline & SB2,7 & $\begin{array}{c}482 \\
498\end{array}$ & 16 & 120 & 480 & 480 & 1 \\
\hline & SB3,8 & $\begin{array}{l}762 \\
778\end{array}$ & 16 & 480 & 360 & 160 & 1 \\
\hline & SB4,9 & $\begin{array}{l}610 \\
626\end{array}$ & 16 & 240 & 240 & 60 & 0.99 \\
\hline & SB5,10 & $\begin{array}{l}2452 \\
2472\end{array}$ & 20 & 360 & 360 & 120 & 0.99 \\
\hline
\end{tabular}

The total number of links $L$ is also varied in the different simulations up to $L=20$ links. Two different types of links are considered in the simulations, Type 1 links with a required bit rate $R_{\text {req, }}=200 \mathrm{Mb} / \mathrm{s}$ and Type 2 links with a required bit rate $R_{\text {req.j }}=100 \mathrm{Mb} / \mathrm{s}$. The link numbers $j$ associated with each type are shown in Table II, where the achievable bit rates $R_{j, i}$ and related rewards $r_{j, S^{(i)}(t)}^{(i)}$ for the different interference states of the considered SBs are also presented. The achievable bit rates are obtained based on the Shannon bound obtained as a function of the signal to noise and interference experienced by each link type. In addition, for $R_{j, i} \geq R_{r e q, j}$, the reward is 1 , whereas for $R_{j, i}<R_{r e q, j}$, the reward follows the formulation in [24]. Each link generates sessions with exponentially distributed durations of average $\bar{D}$ time steps. The time between the end of one session and the beginning of the next will also follow an exponential distribution with average $T_{O F F}$ time steps. $\bar{D}$ and $T_{O F F}$ will be varied in different simulations. Thus, the total session generation rate $\rho$ given by $L /\left(\bar{D}+T_{O F F}\right)$ will also vary. The proposed BBSS framework is configured with $\lambda_{\mathrm{THR}}=$ 0.95 , and $T_{o b s}^{(i)}$ is set to 5 time steps for the HD scenarios and to 120 time steps for the LD scenarios.

TABLE II

CHARACTERISTICS OF THE SBS CONSIDERED IN THE SIMULATIONS

\begin{tabular}{|c|c|c|c|c|c|c|c|c|}
\hline \multirow[b]{2}{*}{ Type } & \multirow[b]{2}{*}{ Link index } & \multirow[b]{2}{*}{ SB } & \multicolumn{2}{|c|}{$\mathbf{S}(\mathbf{i})=\mathbf{0}$} & \multicolumn{2}{|c|}{$S(\mathbf{i})=1$} & \multicolumn{2}{|c|}{$\mathbf{S}(\mathbf{i})=2$} \\
\hline & & & $\begin{array}{c}R_{j, i} \\
(\mathbf{M b} / \mathbf{s})\end{array}$ & $r_{j, 0}^{(i)}$ & $\begin{array}{c}R_{j, i} \\
(\mathbf{M b} / \mathbf{s})\end{array}$ & $r_{j, 1}^{(i)}$ & $\begin{array}{c}R_{j, i} \\
(\mathbf{M} / \mathbf{s})\end{array}$ & $r_{j, 2}^{(i)}$ \\
\hline \multirow{5}{*}{1} & \multirow{5}{*}{$\begin{array}{c}1,4,7,10,13 \\
16,19\end{array}$} & 1,6 & 264 & 1 & 150 & 0.9 & 87 & 0.2 \\
\hline & & 2,7 & 297 & 1 & 193 & 1 & 87 & 0.2 \\
\hline & & 3,8 & 258 & 1 & 152 & 0.9 & 48 & 0 \\
\hline & & 4,9 & 281 & 1 & 175 & 1 & 70 & 0.1 \\
\hline & & 5,10 & 264 & 1 & 69 & 0.1 & 20 & 0 \\
\hline \multirow{5}{*}{2} & \multirow{5}{*}{$\begin{array}{c}2,3,5,6,8,9 \\
11,12,14 \\
15,17,18,20\end{array}$} & 1,6 & 145 & 1 & 40 & 0.2 & 8 & 0 \\
\hline & & 2,7 & 204 & 1 & 98 & 1 & 12 & 0 \\
\hline & & 3,8 & 175 & 1 & 70 & 0.8 & 4 & 0 \\
\hline & & 4,9 & 185 & 1 & 80 & 0.9 & 6 & 0 \\
\hline & & 5,10 & 145 & 1 & 4 & 0 & 0.45 & 0 \\
\hline
\end{tabular}

\section{B. Key Performance Indicators (KPIs)}

The assessment of the proposed framework has been carried out in terms of the following KPIs, selected to account for both the user experience and the efficient operation of the system:

- Average system reward: This is the reward experienced by each data transmission session depending on the interference state of the allocated SB averaged over all links during the total simulation time. The reward ranges between 0 and 1 depending on the bit rate that can be achieved by a radio link in the allocated SB with respect to the required bit rate. Then, it is a metric that directly maps the expected user bit rate requirements with the actual achieved bit rates, i.e. a high reward means that service is being provisioned satisfactorily.

- Average throughput: This is the bit rate in $\mathrm{Mb} / \mathrm{s}$ achieved by the radio links averaged over the total simulation time.

- Observation rate: This is the average number of observations per time step that are performed to determine the interference state of the different SBs during system operation. This metric considers the efficiency of the system operation, i.e. how costly is for the system to acquire the context information from the environment that is used as an input for the spectrum selection decision making. A low observation rate means reducing the measurements taken from the environment and, therefore, results in lower power consumption.

- Blocking probability: This is the probability that a new session must be established for a given link but there are no available SBs.

\section{Performance evaluation of the OSDM selection in the BBSS framework}

To assess the behavior of the OSDM entity that selects the observation strategy in the BBSS framework, this section compares the performance obtained by each of the individual observation strategies and the associated SSDM criteria considered in Section III.B, namely, IM, PM and StS. A set of $M=5 \mathrm{SBs}$ and $L=3$ links (i.e., link numbers $j=1,2,3$ ) has been considered in this case. The 5 different scenarios shown in Table III are considered for this analysis to demonstrate the effect of the observation and decision-making strategies under different situations in terms of interference dynamics and traffic patterns. They are obtained by combining the HD and LD possibilities of interference dynamism in Table I with different values of the average session duration $\bar{L}$, average inactivity period for each link $T_{O F F}$ and total session generation rate $\rho$. Table III indicates the observation strategy that would be selected by the OSDM entity in the proposed BBSS framework for each scenario based on the flow diagram in Figure 3. 
TABLE III

CHARACTERIZATION OF THE SCENARIOS TO ANALYZE THE OSDM BEHAVIOR

\begin{tabular}{|c|c|c|c|c|c|}
\hline Scenario & $\begin{array}{c}\bar{L}_{\text {(time }} \\
\text { steps) }\end{array}$ & $\begin{array}{c}\text { Toff (time } \\
\text { steps) }\end{array}$ & $\boldsymbol{\rho}$ & Dynamicity & $\begin{array}{c}\text { Strategy } \\
\text { selected }\end{array}$ \\
\hline 1 & 3 & 3 & 0.5 & HD & IM \\
\hline 2 & 30 & 1 & 0.097 & & StS \\
\hline 3 & 60 & 320 & $7.8 \cdot 10^{-3}$ & & IM \\
\hline 4 & 30 & 1 & 0.097 & LD & PM \\
\hline 5 & 500 & 60 & $5.3 \cdot 10^{-3}$ & & StS \\
\hline
\end{tabular}

Table IV presents the average reward, throughput and observation rate of the different strategies. The results are obtained as the average for all links during a total simulation time of 100,000 time steps, starting from the time when the $\mathrm{KD}$ statistics were acquired under the considered convergence criterion. The blocking probability is not included in the table because it is 0 in this analysis, as there are always available spectrum blocks.

TABLE IV

AVERAGE PERFORMANCE RESULTS IN TERMS OF REWARD, THROUGHPUT AND OBSERVATION RATE AVERAGED OVER ALL LINKS

\begin{tabular}{|c|c|c|c|c|}
\hline & & Reward & $\begin{array}{c}\text { Throughput } \\
(\mathrm{Mb} / \mathrm{s})\end{array}$ & $\begin{array}{c}\text { Observation Rate } \\
\text { (Observation/Time step) }\end{array}$ \\
\hline \multirow{4}{*}{ Scenario1 } & IM & 0.94 & 123 & 1.94 \\
\hline & PM & 0.84 & 108 & 0.93 \\
\hline & $\mathrm{StS}$ & 0.79 & 107 & 0 \\
\hline & BBSS & 0.94 & 123 & 1.94 \\
\hline \multirow{4}{*}{ Scenario2 } & IM & 0.77 & 100 & 0.3 \\
\hline & PM & 0.75 & 99 & 0.51 \\
\hline & $\mathrm{StS}$ & 0.75 & 100 & 0 \\
\hline & BBSS & 0.75 & 99 & 0 \\
\hline \multirow{4}{*}{ Scenario3 } & IM & 0.94 & 121 & 0.04 \\
\hline & PM & 0.91 & 117 & 0.041 \\
\hline & $\mathrm{StS}$ & 0.84 & 110 & 0 \\
\hline & BBSS & 0.94 & 121 & 0.04 \\
\hline \multirow{4}{*}{ Scenario4 } & IM & 0.85 & 117 & 0.3 \\
\hline & PM & 0.82 & 114 & 0.037 \\
\hline & $\mathrm{StS}$ & 0.73 & 107 & 0 \\
\hline & BBSS & 0.82 & 114 & 0.037 \\
\hline \multirow{4}{*}{ Scenario5 } & IM & 0.78 & 102 & 0.018 \\
\hline & PM & 0.76 & 100 & 0.024 \\
\hline & $\mathrm{StS}$ & 0.76 & 100 & 0 \\
\hline & BBSS & 0.76 & 100 & 0 \\
\hline
\end{tabular}

Focusing on scenario 1, characterized by the high dynamism of the interference together with the short sessions, the results of Table IV highlight that the best performance is achieved by the IM strategy, which is the strategy selected by OSDM in this scenario. In fact, instantaneous measurements allow improvements of approximately $12 \%$ and $19 \%$ with respect to the $\mathrm{PM}$ and $\mathrm{StS}$ solutions, respectively, to be obtained. In scenario 2, the interference dynamics are the same as in scenario 1, but longer sessions are considered. Correspondingly, the interference will exhibit variations over the session duration, meaning that the performance is not highly sensitive to the actual interference state at the time when a session starts. Instead, the steady-state probabilities are generally representative of the actual interference conditions that will be experienced during each session. Table IV illustrates that in this scenario, there are small differences in the reward performance obtained by IM, PM and StS; thus, because StS does not require observations to be carried out, it is the best choice.

Scenario 3 is characterized by less dynamic interference. Moreover, the session duration is such that $\bar{D}<-1 / \ln \left|\lambda_{1}^{(i)}\right|$ and the session generation rate is low, meaning that in this case, the IM approach is the one selected by the proposed BBSS framework. The results in Table IV reveal that this is a suitable option because it allows the highest reward to be obtained with greatly reduced observation requirements. Scenario 4 has the same interference dynamics and session duration as scenario 3 but a higher session generation rate. As a result, IM has an observation rate that is nearly 7.5 times higher than that of scenario 3. Therefore, the PM strategy becomes a better option because it achieves a similar reward to IM (the difference is less than 3.5\%) but with less observation, as well as an improvement of approximately $12 \%$ with respect to StS. Correspondingly, the selection made by OSDM in this scenario is PM.

Finally, scenario 5 is characterized by the same interference dynamics as in scenarios 3 and 4, but now, the session duration is considerably longer; thus, in this case, OSDM selects StS as the most appropriate strategy. The results in Table IV reveal that this is the best option because it provides nearly the same reward performance as IM and PM but without requiring observations.

\section{Benchmarking}

To benchmark the performance of the proposed BBSS framework, two strategies presented in the literature have been implemented in the simulation framework described above and are compared with the BBSS solution. In particular, the channel capacity-based (CCB) spectrum selection algorithm presented in [8] and the selective opportunistic spectrum access (SOSA) algorithm presented in [9] have been considered as representative techniques that address the same problem considered in this paper and enable the establishment of a comparison under the same set of assumptions. The details of the implementation of both algorithms in the simulation framework are given in Appendix 3. Additionally, as a baseline reference, a random strategy in which the SB is randomly selected among those available at the time that each session is established is also included in the comparison.

Figure 4 presents the performance achieved by the proposed BBSS framework and by the reference SOSA, CCB and random strategies as a function of the number of radio links $L$ for the configuration of SBs according to the HD case. The analysis considers $\mathrm{M}=10 \mathrm{SBs}$. For each link, the average duration of the generated sessions and inactivity periods are $\bar{D}=3$ time steps and $T_{O F F}=3$ time steps, respectively. The performance in terms of reward is depicted in Figure 4(a), where it can be observed that the proposed BBSS approach outperforms all the other strategies. In particular, it outperforms SOSA in around $4 \%, \mathrm{CCB}$ in around $26 \%$ and the baseline random case in around $53 \%$. It is worth mentioning that, based on the interference and traffic dynamics existing in this analysis, the OSDM entity of BBSS is selecting the IM 
observation strategy. The performance in terms of observation rate is plot in Figure 4(b), where it can be observed that both $\mathrm{CCB}$ and SOSA require a much higher observation rate than the proposed BBSS, because both strategies need to measure all the SBs in each time step to keep track of the interference dynamics. The blocking probability, which is the same for all the strategies, is plot in Figure 4(c). It can be noticed how it starts to increase when the number of links $\mathrm{L}$ is higher than the number of spectrum blocks $M=10$, because in this case it may happen that all the SBs are already allocated to other links at the time of starting a new session, so the new session will be blocked.

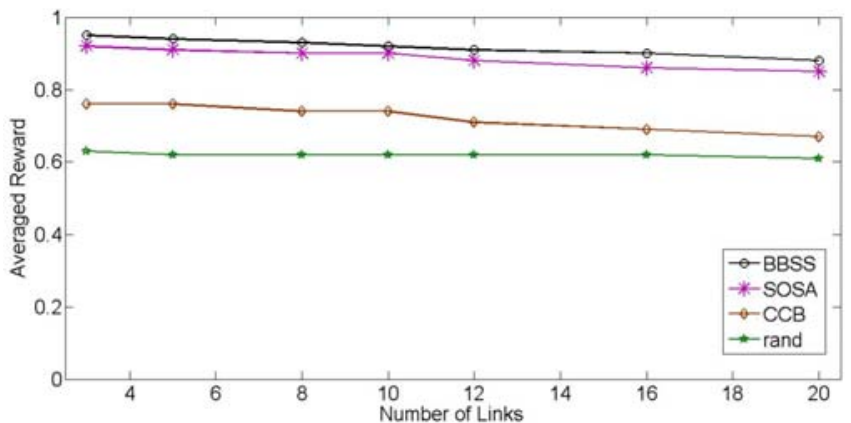

(a)

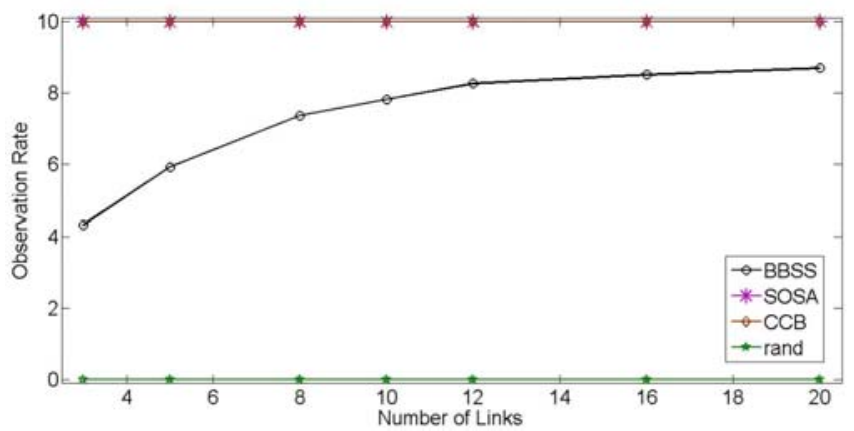

(b)

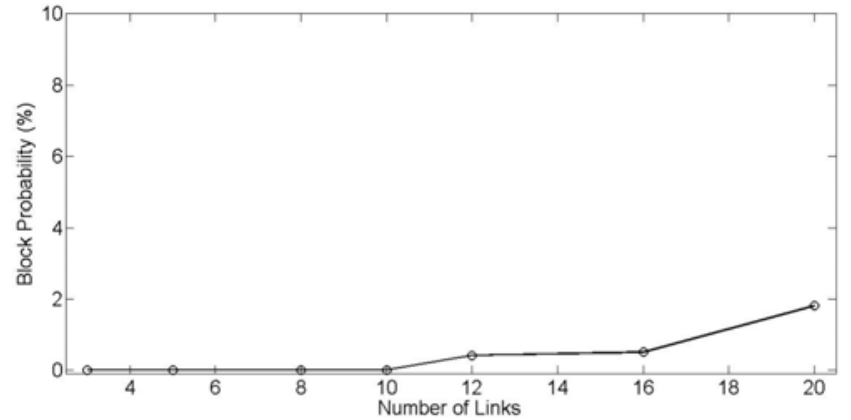

(c)

Fig. 4 Comparative performance between the proposed framework and reference strategies from the literature in an HD scenario in terms of (a) reward, (b) observation rate, and (c) blocking probability.

Figure 5 analyses the achieved performance for the LD case, when the interference variations are less dynamic. In this case, the average duration of the generated sessions and inactivity periods for the different links are $\bar{L}=60$ time steps and $T_{O F F}=320$ time steps, respectively. Based on the interference and traffic conditions existing in this case, the OSDM entity selects the IM strategy when the number of links
$\mathrm{L}$ is less than 4 (and correspondingly, the total session generation rate $\rho$ is lower than $1 / T_{d b s}^{(i)}$ ) and the PM strategy when the number of links $\mathrm{L}$ is greater than or equal to 4 . Figure 5(a) illustrates that the proposed BBSS approach offers a significantly better performance in terms of reward than both the $\mathrm{CCB}$ and random approaches. BBSS and SOSA offer similar behaviors in terms of reward, with a slightly better performance for SOSA, particularly when the number of links is high. However, in terms of the observation rate, as shown in Figure 5(b), BBSS requires many fewer observations than SOSA; thus, the proposed BBSS framework offers a better trade-off between reward performance and observation rate requirements.

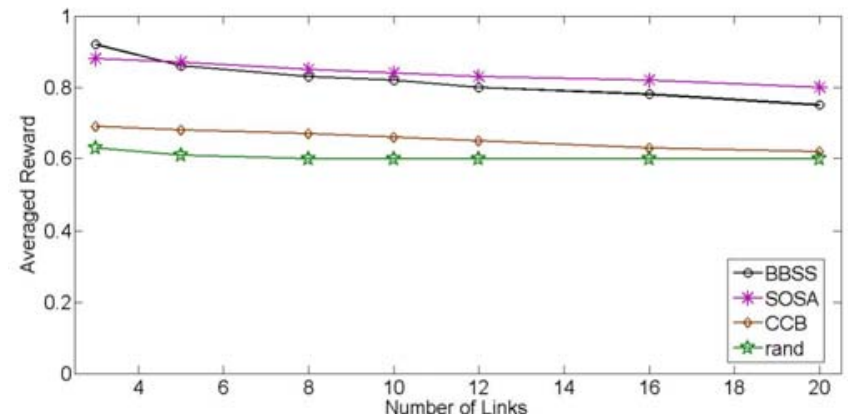

(a)

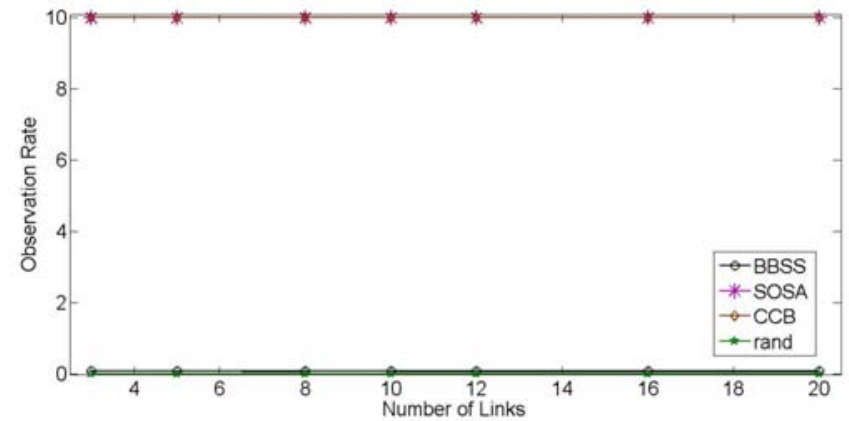

(b)

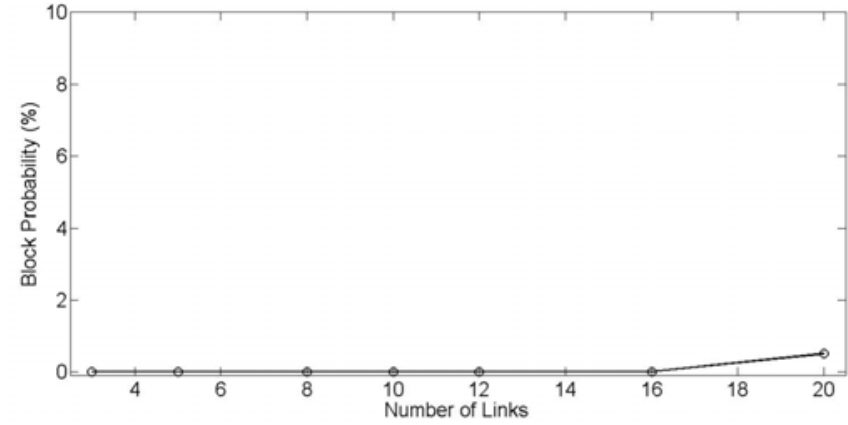

(c)

Fig. 5 Comparative performance between the proposed framework and reference strategies from the literature in an LD scenario in terms of (a) reward, (b) observation rate, and (c) blocking probability.

\section{E. Experimental validation}

For further validation, the proposed BBSS cognitive management framework described in Section III has been implemented in a real-time testbed platform consisting of six reconfigurable universal software radio peripheral (USRP) 
nodes that allow for an evaluation of a scenario with one radio link and $M=3 \mathrm{SBs}$ affected by variable interference conditions. The specific details of the testbed architecture, implementation and configuration are the same as in [25].

The testbed has been used to validate practical aspects related to the dynamic variation of the interference sources, which may differ from the theoretical assumptions made by the model. In this respect, Table $\mathrm{V}$ presents the comparison in terms of the reward achieved by the proposed BBSS framework when the interference states behave as a discretetime Markov process, as assumed by the system model, and when the duration of the interference states is not Markovian but follows a uniform distribution with the same average. In this latter case, the BBSS framework operates by performing the same computations as if the interference was Markovian. The reduction in terms of the reward associated with the interference not exhibiting Markovian property is small, i.e., less than $3.1 \%$ in all analyzed scenarios.

TABLE V

EFFECT OF THE STATISTICAL PATTERN OF THE INTERFERENCE ON THE REWARD FOR DIFFERENT SCENARIOS

\begin{tabular}{|c|c|c|}
\hline \\
\hline & $\begin{array}{l}\text { Markovian } \\
\text { interference }\end{array}$ & $\begin{array}{c}\text { Non-Markovian } \\
\text { interference }\end{array}$ \\
\hline $\begin{array}{l}\text { Scenario A: average durations for } \\
\text { SB } 1,2,3: S^{(i)}=0\{24,3,24\} ; S^{(i)}=1 \\
\{6,24,8\}, \overline{D_{j}}=1, \rho=0.5 \\
\text { sessions/time step; }\end{array}$ & 0.94 & $0.91(-3.1 \%)$ \\
\hline $\begin{array}{l}\text { Scenario B: average durations for } \\
\text { SB } 1,2,3: S^{(i)}=0\{24,3,24\} ; S^{(i)}=1 \\
\{6,24,8\}, \overline{D_{j}}=15, \rho=0.063 \\
\quad \text { sessions/time step; }\end{array}$ & 0.86 & $0.84(-2.3 \%)$ \\
\hline $\begin{array}{l}\text { Scenario C: average durations for } \\
\text { SB } 1,2,3: S^{(i)}=0\{480,60,480\} ; \\
S^{(i)}=1\{120,480,160\}, \overline{D_{j}}=15, \\
\rho=0.013 \text { sessions/time step; }\end{array}$ & 0.97 & $0.95(-2.1 \%)$ \\
\hline
\end{tabular}

\section{CONCLUSIONS}

This paper has proposed a comprehensive cognitive management framework for spectrum selection in cognitive radio networks. The proposed functional model incorporates a generic formulation of the spectrum selection in terms of the belief vector concept, which characterizes and predicts the dynamics of the interference affecting a given radio environment.

The proposed framework relies on a knowledge management entity that extracts the relevant knowledge from the radio environment. In this respect, the paper has shown that the interference dynamics in the spectrum blocks can be properly captured by the second highest eigenvalue of the state transition matrix. Therefore, this metric has been proposed as a key driver to jointly select the observation strategy and the spectrum selection decision-making criterion to properly balance the trade-off between achievable performance and measurement requirements.

The proposed framework has been evaluated via simulations in different scenarios. Evaluation has been done in terms of both the performance obtained by the users, captured in the reward metric that directly maps the expected user bit rate requirements with the actual achieved bit rates, and the operational implications for the system, captured in terms of the observation rate requirements. Results have illustrated the capability of the proposed framework to select the observation strategy that best matches the trade-off between reward and observation rate depending on the characteristics of the scenario. The proposed approach has also been compared against other state-of-the-art solutions, revealing that it can achieve similar or even better performance in terms of reward with less stringent observation requirements. Finally, a testbed has been used to validate the robustness of the proposed approach in front of non-Markovian interference dynamics, revealing reward degradations of less than $3 \%$.

\section{APPENDIX 1- ProOF OF THEOREM 1}

Let us consider the eigenvalue decomposition of matrix $\mathbf{P}^{(\mathbf{i}) \mathbf{T}}$ :

$\mathbf{P}^{(\mathbf{i}) \mathbf{T}}=\mathbf{V} \cdot \mathbf{\Sigma} \cdot \mathbf{V}^{-1}$

where $\boldsymbol{\Sigma}=\operatorname{diag}\left(\lambda_{0}^{(i)}, \lambda_{1}^{(i)}, \ldots, \lambda_{K}^{(i)}\right)$ is a diagonal matrix formed by the ordered eigenvalues of $\mathbf{P}^{(\mathbf{i}) \mathbf{T}}\left|\lambda_{0}^{(i)}\right| \geq\left|\lambda_{1}^{(i)}\right| \geq \ldots \geq\left|\lambda_{K}^{(i)}\right|$. The eigenvalues of $\mathbf{P}^{(\mathbf{i}) \mathbf{T}}$ are the same as those of $\mathbf{P}^{(\mathbf{i})}$. Because $\mathbf{P}^{(\mathbf{i})}$ is a stochastic irreducible matrix, the Perron-Frobenius theorem [33] ensures that the largest eigenvalue is unique and equal to $\lambda_{0}^{(i)}=1$ such that $\left|\lambda_{k}^{(i)}\right|<1$ for $k>0$. Moreover, $\mathbf{V}=\left[\mathbf{v}_{\mathbf{0}} \mathbf{v} \mathbf{1} \ldots \mathbf{v}_{\mathbf{K}}\right]$ is a matrix whose columns are the eigenvectors of $\mathbf{P}^{(\mathbf{i}) \mathbf{T}}$. By multiplying $n$ times (23), the eigenvalue decomposition of matrix $\left[\mathbf{P}^{(\mathbf{i}) \mathrm{T}}\right]^{n}$ is easily obtained as

$\left[\mathbf{P}^{(\mathbf{i}) \mathbf{T}}\right]^{n}=\mathbf{V} \cdot \mathbf{\Sigma}^{n} \cdot \mathbf{V}^{-1}$

where $\boldsymbol{\Sigma}^{n}=\operatorname{diag}\left(\left[\lambda_{0}^{(i)}\right]^{n},\left[\lambda_{1}^{(i)}\right]^{n}, \ldots,\left[\lambda_{K}^{(i)}\right]^{n}\right)$. Then, the

following relationship is fulfilled:

$\left[\mathbf{P}^{(\mathbf{i}) \mathbf{T}}\right]^{n} \cdot \mathbf{v}_{\mathbf{k}}=\left[\lambda_{k}^{(i)}\right]^{n} \mathbf{v}_{\mathbf{k}}$

or, by transposing both sides of the equation,

$\mathbf{v}_{\mathbf{k}}^{\mathbf{T}} \cdot\left[\mathbf{P}^{(\mathbf{i})}\right]^{n}=\left[\lambda_{k}^{(i)}\right]^{n} \mathbf{v}_{\mathbf{k}}^{\mathbf{T}}$

In addition, the steady state probability vector $\pi^{(i)}$ of an ergodic discrete time Markov process fulfills the following relationship [31]:

$\boldsymbol{\pi}^{(\mathrm{i}) \mathrm{T}} \mathbf{P}^{(\mathrm{i})}=\boldsymbol{\pi}^{(\mathrm{i}) \mathrm{T}}$

A comparison of (26) with (27) demonstrates that for the case $\mathrm{n}=0$, the steady-state probability vector is the eigenvector associated with $\lambda_{0}^{(i)}=1$, that is,

$\mathbf{v}_{0}^{\mathbf{T}}=\boldsymbol{\pi}^{(\mathbf{i}) \mathbf{T}}$

Moreover, because the eigenvectors $\mathbf{v}_{\mathbf{k}}^{\mathbf{T}}$ are orthonormal, any arbitrary vector and, in particular, the belief vector $\mathbf{b}^{(\mathbf{i}) \mathbf{T}}(t)$, can be expressed as a linear combination of these eigenvectors, that is 


$$
\mathbf{b}^{(\mathbf{i}) \mathbf{T}}(t)=\sum_{k=0}^{K} a_{k} \mathbf{v}_{\mathbf{k}}^{\mathbf{T}}
$$

Consequently, combining (14), (26), (28), and (29) yields

$\mathbf{b}^{(\mathbf{i}) \mathbf{T}}(t+n)=\sum_{k=0}^{K} a_{k} \mathbf{v}_{\mathbf{k}}^{\mathbf{T}} \cdot\left[\mathbf{P}^{(\mathbf{i})}\right]^{n}=\sum_{k=0}^{K} a_{k}\left[\lambda_{k}^{(i)}\right]^{n} \mathbf{v}_{\mathbf{k}}^{\mathbf{T}}=a_{0} \pi^{(\mathbf{i}) \mathbf{T}}+\sum_{k=1}^{K} a_{k}\left[\lambda_{k}^{(i)}\right]^{n} \mathbf{v}_{\mathbf{k}}^{\mathbf{T}}(30$

Because $\left|\lambda_{k}^{(i)}\right|<1$ for all $k>0$, the second term of the summation in (30) will tend to 0 when $n \rightarrow \infty$, so the belief vector $\mathbf{b}^{(\mathbf{i}) \mathbf{T}}(t+n) \rightarrow a_{0} \pi^{(\mathbf{i}) \mathbf{T}}=\pi^{(\mathbf{i}) \mathbf{T}}$ tends to the steady state. $a_{0}=1$ because otherwise, the summation of all components of $\mathbf{b}^{(\mathbf{i}) \mathbf{T}}(t+n)$ (i.e., the sum of the probabilities for all states) would not be 1 . Moreover, considering the summation in (30), the speed of convergence will be driven by the largest value of $\left|\lambda_{k}^{(i)}\right|^{n}, k>0$ or, equivalently, by the absolute value of the largest eigenvalue after $\lambda_{0}^{(i)}=1$, that is $\left|\lambda_{1}^{(i)}\right|$. A lower value of $\left|\lambda_{1}^{(i)}\right|$ results in a more rapid convergence, reflecting that the scenario suffers from more variability.

\section{APPENDIX 2 - PROOF OF COROLLARY 1}

The dynamic evolution of the belief vector $\mathbf{b}^{(\mathbf{i}) \mathbf{T}}(t)$ toward the steady state is reflected in (30) for $n \rightarrow \infty$. The speed of the convergence is driven by the term $\left[\lambda_{1}^{(i)}\right]^{n}$, whose absolute value can be expressed as

$\left|\lambda_{1}^{(i)}\right|^{n}=e^{n \cdot \ln \left|\lambda_{1}^{(i)}\right|}$

When increasing $n$, this relationship is a classical exponential decay with time constant $\tau=-1 / \ln \left|\lambda_{1}^{(i)}\right|$ time steps. Then, the value $-1 / \ln \left|\lambda_{1}^{(i)}\right|$ provides a rule of thumb to estimate the time needed for the convergence of the belief vector $\mathbf{b}^{(\mathbf{i}) \mathrm{T}}(t)$.

\section{APPENDIX 3 - PROOF OF COROLLARY 1}

\section{A. Channel capacity-based (CCB) allocation algorithm}

Based on [8], when the spectrum selection is triggered, this algorithm determines the SB to be allocated based on the expected time that each SB will remain in the idle state (i.e., state $S^{(i)}=0$ in our framework) and on the bit rate achievable in each SB. For that purpose, the algorithm must observe all SBs in every time step to detect the time when each SB enters the state $S^{(i)}(t)=0$ and to keep track of the number of time steps since the $i$-th SB entered $S^{(i)}(t)=0$, denoted as $N_{o, i}$, and the average duration of the state $S^{(i)}(t)=0$, denoted as $1 / \mu_{i}$ and measured in time steps. Then, each time $t$ that a new session is established for link $j$, the spectrum selection carries out the process explained in Algorithm 1 among the SBs in state $S^{(i)}(t)=0$. If no $\mathrm{SB}$ is found in state $S^{(i)}=0$, the $\mathrm{SB}$ with the lowest value of $\mu_{i}$ ' is allocated. This last condition is included here to provide a fair comparison with the proposed BBSS framework, which allows the allocation of SBs even if they are not in state $S^{(i)}=0$. Similarly, to have a fair comparison, the algorithm in [8] has been particularized to the case where only one channel can be allocated to one link.

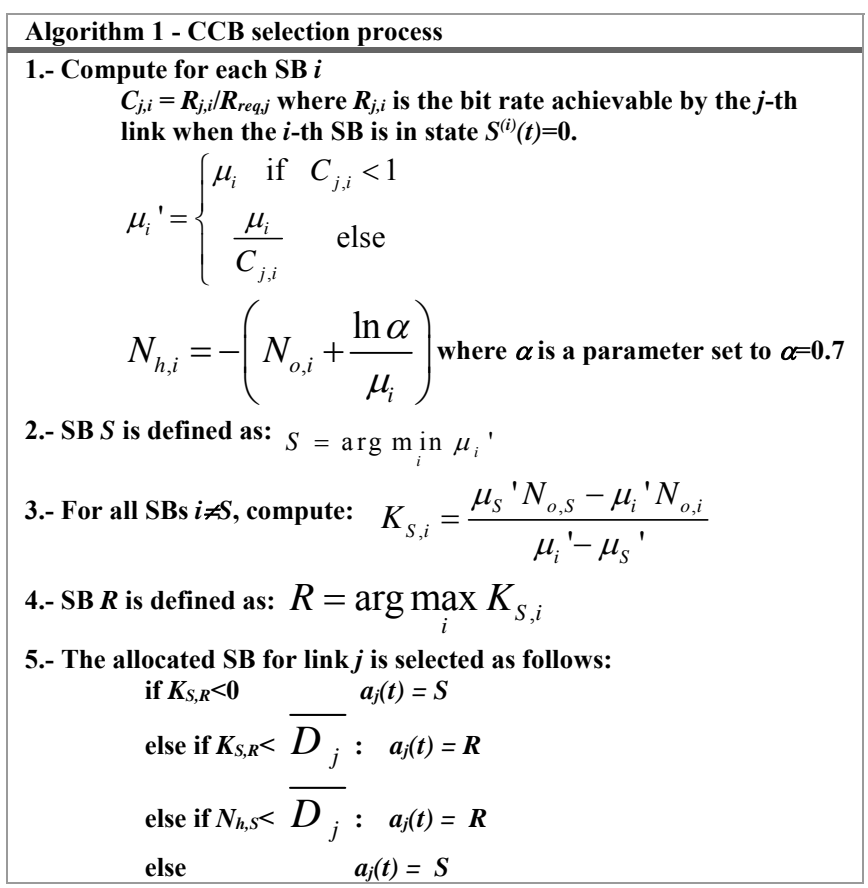

B. Selective opportunistic spectrum access (SOSA) algorithm

As explained in [9], this algorithm predicts the probability that a SB is idle (i.e., in state $S^{(i)}=0$ in our framework) for the next time step based on previous long-term measurements of the idle and active periods. Then, in each time step, it measures all SBs, and based on the result, it updates the probability $p_{i}$ that the $i$-th SB is in state $S^{(i)}=0$. Then, each time that a new session is established for link $j$, the spectrum selection process just measures the SBs in decreasing order $p_{i}$ and assigns the first $\mathrm{SB}$ that is found in state $S^{(i)}=0$. If no SB is found in state $S^{(i)}=0$, the one with the highest $p_{i}$ is assigned. This last condition has been included here to provide a fair comparison with the proposed BBSS framework, which allows the allocation of SBs even if they are not in state $S^{(i)}=0$.

\section{ACKNOWLEDGMENT}

The work is supported by the Spanish Research Council and FEDER funds under RAMSES grant (ref. TEC2013-41698$\mathrm{R})$.

\section{REFERENCES}

[1] J. Mitola III, "Cognitive radio: an integrated agent architecture for software defined radio," Ph.D. dissertation, KTH Royal Institute of Technology, Stockholm, Sweden, 2000.

[2] IEEE Std 1900.1TM-2008, "IEEE Standard Definitions and Concepts for Dynamic Spectrum Access: Terminology Relating to Emerging Wireless Networks, System Functionality, and Spectrum Management".

[3] I.F. Akyildiz, et al. "Next generation/dynamic spectrum access/cognitive radio wireless networks: a survey", Computer Networks, Sep. 2006, Vol. 50, Issue 13, pp. 2127-2159. 
[4] O. Sallent, et al. "A Roadmap from UMTS Optimization to LTE SelfOptimization”, IEEE Communications Magazine, Jun. 2011, Vol.49, Issue 6, pp. 172-182.

[5] P. Demestichas, et al., "5G on the Horizon. Key Challenges for the Radio-Access Network", IEEE Vehicular Technology Magazine, Sep. 2013, Vol. 8, Issue 3, pp. 47-53.

[6] ETSI TR 102682 v1.1.1, "Reconfigurable Radio Systems (RRS); Functional Architecture (FA) for the Management and the Control of Reconfigurable Radio Systems", July 2009.

[7] V. Stavroulaki, et al. "Cognitive Control Channels: From Concept to Identification of Implementation Options", IEEE Communications Magazine, Jul. 2012, Vol. 50, Issue 7, pp. 96-108.

[8] J. Lee, H-K. Park, "Channel Prediction-Based Channel Allocation Scheme for Multichannel Cognitive Radio Networks", Journal of Communications and Networks, Apr. 2014, Vol. 16, Issue 2, pp. 209216.

[9] G. Yuan, R. C. Grammenos, Y. Yang, W. Wang, "Performance Analysis of Selective Opportunistic Spectrum Access With Traffic Prediction", IEEE Transactions on Vehicular Technology, May 2010, Vol. 59, Issue 4, pp. 1949-1959.

[10] X. Xing, T. Jing, W. Cheng, Y. Huo, X. Cheng, "Spectrum Prediction in Cognitive Radio Networks", IEEE Wireless Communications, Apr 2013, Vol. 20, Issue 2, pp. 90 - 96.

[11] V. K. Tumuluru, P. Wang, D. Niyato, "Channel status prediction for cognitive radio networks", Wireless Communications and Mobile Computing, Jun. 2012, Vol. 12, Issue 10, pp. 862-874.

[12] P. Huang, et al., "Wireless Spectrum Occupancy Prediction based on Partial Periodic Pattern Mining", IEEE Trans. on Parallel and Distrib. Systems, Jul. 2014 Vol. 25, Issue 7, pp. 1925-1934.

[13] H. Ahmadi, I. Macaluso, L.A. DaSilva, "Carrier Aggregation as a Repeated Game: Learning Algorithms for Efficient Convergence to a Nash Equilibrium", GLOBECOM 2013, Atlanta, Georgia, USA, Dec. 2013.

[14] Y. Xu, et al. "Decision-Theoretic Distributed Channel Selection for Opportunistic Spectrum Access: Strategies, Challenges and Solutions", IEEE Comms Surveys and Tutorials, Fourth Quarter, 2013, Vol. 15, Issue 4, pp. 1689-1713.

[15] K.P. Murphy, "A Survey of POMDP Solution Techniques", available at http://http.cs.berkeley.edu/ murphyk/Papers/pomdp.ps.gz, 2000.

[16] Q. Zhao, et al. "Decentralized Cognitive MAC for Opportunistic Spectrum Access in Ad Hoc Networks: A POMDP Framework", IEEE Jour. on Sel. Areas in Comms., Apr. 2007, Vol.25, Issue 3, pp. 589-600.

[17] H. Li, X. Feng, X. Gan, Z. Cao, "Joint Specrtum Sensing and Transmission Strategy for Energy-Efficient Cognitive Radio Networks", CROWNCOM 2013, Washington DC, USA, Jul. 2013.

[18] H. Liu, B. Krishnamachari, Q. Zhao, "Cooperation and Learning in Multiuser Opportunistic Spectrum Access”, ICC 2008, Beijing, China, May 2008.

[19] A. Raschellà et al. "On the use of POMDP for Spectrum Selection in Cognitive Radio Networks", CROWNCOM 2013, Washington DC, USA, Jul. 2013

[20] A. Raschellà, et al. "On the impact of the Observation Strategy in a POMDP-based framework for Spectrum Selection", PIMRC 2013, London, UK, Sep. 2013.

[21] K. Wang, et al. "On Optimality of Myopic Sensing Policy with Imperfect Sensing in Multi-Channel Opportunistic Access", IEEE Trans. on Comms, Sep. 2013, Vol. 61, Issue 9, pp. 3854-3862.

[22] W. Jouini, C. Moy, J. Palicot, "Decision making for cognitive radio equipment: analysis of the first 10 years of exploration", EURASIP Journ. on Wireless Comms. and Netw., Dec. 2012, 2012:26, pp. 1-16.

[23] O. Altrad, et al. "Opportunistic Spectrum Access in Cognitive Radio Networks under Imperfect Spectrum Sensing", IEEE Transactions on Vehicular Technology, Feb. 2014, Vol. 63, Issue 2, 920-925.

[24] J. Pérez-Romero, et al. "Enhanced Cognitive Radio Operation through Belief-based Decision Making”, EW 2014, Barcelona, Spain, May 2014.

[25] A. Raschellà, et al. "Evaluation of a Belief-based Decision Making in a Real-time Platform for Cognitive Radio Networks", CROWNCOM 2014, Oulu, Finland, Jun. 2014.

[26] R. Ferrus, et al. "A Solution Framework to provide Management Services for Wireless Communications in the Digital Home", IEEE Comms. Magazine, Nov. 2012, Vol.50, Issue 12, pp. 132-141.
[27] F. M. Abinader, et al. "Enabling the Coexistence of LTE and Wi-Fi in Unlicensed Bands", IEEE Communications Magazine, Nov. 2014, Vol.52, Issue 11, pp. 54-61.

[28] S. Andreev, et al. "Analyzing Assisted Offloading of Cellular User Sessions onto D2D Links in Unlicensed Bands", IEEE Jour. on Sel. Areas in Comms., Jan. 2015, Vol. 33, Issue 1, pp. 67-80.

[29] ETSI EN 301893 v1.7.2 "Broadband Radio Access Networks (BRAN): $5 \mathrm{GHz}$ high performance RLAN; Harmonized EN covering the essential requirements of article 3.2 of the R\&TTE Directive", Jul. 2014.

[30] F. Bouali, et al. "Exploiting Knowledge Management for Supporting Spectrum Selection in Cognitive Radio Networks", CROWNCOM 2012, Stockholm, Sweden, Jun. 2012.

[31] L. Kleinrock, Queueing Systems. Volume I: Theory, John Wiley \& Sons, 1975.

[32] F. Bouali, et al. "Exploiting Knowledge Management for Supporting Multi-Band Spectrum Selection in Non-Stationary Environments", IEEE Trans. on Wireless Comms, Dec. 2013, Vol. 12, Issue 12, pp. 62286243.

[33] C.D.Meyer, Matrix Analysis and Applied Linear Algebra Book ad Solutions Manual, Society for Industrial and Applied Mathematics, 2000.

Jordi Pérez-Romero is associate professor at the Dept. of Signal Theory and Communications of the Universitat Politècnica de Catalunya (UPC) in Barcelona, Spain. He received the Telecommunications Engineering degree and the Ph.D. from the same university in 1997 and 2001, respectively. Since then he has been actively working in the field of mobile and wireless communication systems, with particular focus on packet radio techniques, radio resource and QoS management, heterogeneous wireless networks, cognitive radio networks, self-organized networks and network optimization. He has been involved in different European Projects such as EVEREST, E2R, NEWCOM, AROMA, E3, FARAMIR, OneFIT, SESAME with different responsibilities as researcher, WP leader and UPC project responsible. He has also participated in different projects for private companies. He has published more than 200 papers in international journals and conferences and has co-authored three books and contributed to 7 book chapters. He holds two international patents and has contributed to 3GPP and ETSI standardization bodies. He is associate editor of two international journals (IEEE Vehicular Technology Magazine and EURASIP Journal on Wireless Communications Networks) and has been TPC in different international conferences. Besides, he participated in the organization of IEEE VTC in Spring 2009 and IEEE PIMRC 2004 international conferences, hold in Barcelona. He has also been TPC Co-chair of EuCNC conference 2014 and of VTC Spring 2015. Moreover, he has organized several workshops, special sessions and special issues in international conferences and journals.

Alessandro Raschellà received the M.Sc. in Telecommunications Engineering from the University Mediterranea of Reggio Calabria (UNIRC), Italy in 2007 and the Ph.D. degree in Wireless Communications from the Universitat Politècnica de Catalunya (UPC), Barcelona, Spain in 2015. From 2007 to 2009, he was a Research Assistant of the Department of Information Engineering, of Infrastructures and Sustainable Energy (DIIES) of the UNIRC. He is currently a Postdoctoral Research Associate at the School of Computing \& Mathematical Sciences of the Liverpool John Moores University (LJMU), UK. His research interests 
include Dynamic spectrum access and management, Wireless networks optimization, Cognitive radio networks, Softwaredefined networking.

Oriol Sallent is a Professor at the Universitat Politècnica de Catalunya (UPC). He has participated in a wide range of european projects with diverse responsibilities as WPLeader and Coordinator partner and contributed to standardisation bodies such as 3GPP, IEEE and ETSI. He has published 200+ papers mostly in IEEE journals and conferences. His research interests include, among others, Cognitive management in cognitive radio networks, Dynamic spectrum access and management, Self-organising networks and radio network optimization, Integration of satellite and terrestrial networks and QoS provisioning in heterogeneous wireless networks.

Anna Umbert received the Telecommunications Engineering degree from the Universitat Politècnica de Catalunya (UPC) in 1998, and the Ph.D. degree from the same university in 2004. In 2001 she joined UPC as an assistant professor at the Signal Theory and Communications Department, which is her current status. Her research interest is focused in radio resource and
QoS management in the context of heterogeneous wireless networks, cognitive management in cognitive radio networks, dynamic spectrum access and management, self-organized networks and network optimization. Since 1997 she has participated in several projects founded by both public and private organizations. In particular, she participated in the European Projects RAINBOW, WINEGLAS, CAUTION++, ARROWS, EVEREST, AROMA, OneFIT, FARAMIR and the Networks of Excellence NEWCOM, NEWCOM++ and NEWCOM\#. Currently she is participating in SESAME. She has published more than 50 papers in international journals and conferences. Besides, she participated in the organization of IEEE VTC in Spring 2009 and IEEE PIMRC 2004 international conferences, hold in Barcelona. 